\title{
ResearchOnline@JCU
}

This is the Accepted Version of a paper published in the

Journal: Applied Physiology, Nutrition and Metabolism

Young, Fiona L.S., and Leicht, Anthony S. (2011) Short-term stability of resting heart rate variability: influence of position and gender. Applied Physiology, Nutrition and Metabolism, 36 (2). pp. 210-218.

http://dx.doi.org/10.1139/H10-103

(C) 2015. This manuscript version is made available under the CC-BY-NC-ND 4.0 license

http://creativecommons.org/licenses/by-nc-nd/4.0/

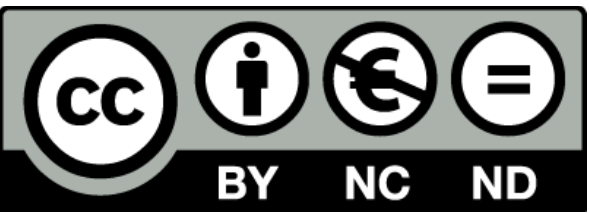




\section{Short-term stability of resting heart rate variability: Influence of position and gender}

Fiona L. S. Young and Anthony S. Leicht

Correspondence and proofs to:

Associate Professor Anthony Leicht

Institute of Sport and Exercise Science

James Cook University

Townsville, Queensland, 4811, AUSTRALIA.

Telephone: \pm 61747814576

Facsimile: \pm 61747816688

E-mail: Anthony.Leicht@jcu.edu.au

Fiona L. S. Young

Institute of Sport and Exercise Science, James Cook University, Townsville, Queensland, 4811

AUSTRALIA (Fiona.Young@jcu.edu.au)

Anthony S. Leicht

Institute of Sport and Exercise Science, James Cook University, Townsville, Queensland, 4811

AUSTRALIA (Anthony.Leicht@jcu.edu.au) 


\begin{abstract}
Heart rate variability (HRV) is utilised within laboratory and clinical settings as a non-invasive indicator of cardiac autonomic modulation. Past research has utilised a wide variety of resting methodologies, and as such, it is difficult to draw conclusions on the nature of HRV from different studies. Therefore, the current study aimed to assess the short-term stability of resting HRV during a 40-minute resting trial and the impact of body position and gender on this short-term stability. Resting HRV was determined from 40-minute trials in three standard positions (supine, seated, and standing) for healthy males $(n=14)$ and females $(n=16)$. Time-domain, geometric, and frequency-domain measures of resting HRV were examined during consecutive 10-minute segments using a three-way ANOVA (time $\mathrm{x}$ position $\mathrm{x}$ gender) and Tukeys' post-hoc tests with reproducibility assessed by intraclass correlation coefficients (ICC) and coefficients of variation. During rest, most HRV measures: fluctuated over time; were greater in the supine compared to the standing position; and were greater for males compared to females. Variables that reflected primarily vagal modulations of heart rate remained stable whereas other HRV measures varied over time. The majority of HRV variables exhibited substantial to excellent short-term reproducibility (ICC >0.6) with time-domain and geometric measures of HRV demonstrating greater values compared to frequency-domain parameters. Based on the current results, the recording and analysis of HRV at 0-10 minutes of rest was recommended as a standardised protocol for the assessment of resting HRV in any standard position for either gender during laboratory and/or clinical settings.
\end{abstract}

Keywords: autonomic control, heart rate, gender, resting position, reproducibility, parasympathetic 


\section{Introduction}

Heart rate variability (HRV) has been investigated in a variety of settings including rest, exercise and various pathologies (Aubert et al. 2001; Kleiger et al. 1987; Leicht et al. 2003b; Task Force of the European Society of Cardiology and the North American Society of Pacing and Electrophysiology 1996). Most importantly, lower HRV has been associated with poor clinical outcome and increased morbidity and mortality (Tsuji et al. 1994), with HRV utilised as a prognostic and diagnostic clinical tool for assessing the autonomic modulation of heart rate (Fei et al. 1996). The significant clinical value of HRV in a wide range of pathologies has led to subsequent investigation of the stability and reproducibility of HRV parameters.

The majority of research investigating HRV stability or reproducibility has focussed on the long-term reproducibility; that is, the perturbations in HRV trials separated by days, weeks, months, and years. These studies have determined that, in general, HRV is reproducible over the long-term (days, weeks, years) (Tarkiainen et al. 2005; Van Hoogenhuyze et al. 1991). Marks and Lightfoot (1999) investigated the long-term reproducibility of 2.5 and 5-minute recordings of HRV obtained from two trials within a one week period and determined that all time-domain variables were highly reproducible (ICC $\geq 0.84$ ), while frequency-domain variables were less reproducible (ICC range 0.67-0.96). These investigators also determined that recording length did not significantly alter any of the investigated time-domain variables or some of the spectral measure of HRV (Marks and Lightfoot 1999). In a review of HRV literature, HRV measures were shown to be moderately reproducible in a variety of resting situations, and that the duration of time between trials had little effect on reproducibility (Sandercock et al. 2005). However, these findings were obtained in different population groups and with different resting protocols, leading Sandercock and colleagues (2005) to suggest that researchers needed to reconsider the available literature with regards to protocol, equipment, data analysis and population.

To date, most studies investigating the reproducibility or stability of short-term measures of HRV have utilised the same protocol in each trial for comparison. While this shows good scientific practice, most studies have conducted resting protocols of differing preparation and recording times, which has made comparisons between studies difficult (Leicht et al. 2003b; Sandercock et al. 2004; Tulppo et al. 1998; Yamamoto et al. 1991). While HRV may be stable from day to day, and week to week, less is known about the within-recording stability. Identification of this within-recording stability may allow for accurate comparisons between different populations and studies.

Freed and colleagues (Freed et al. 1994) investigated two adjacent 10-minute recordings in patients prior to, and 10-hours following reconstructive knee surgery and determined that the frequency-domain parameters obtained were highly reproducible (correlation coefficients $\geq 0.74$; coefficient of variation $[\mathrm{CV}] \leq 15 \%$ ). However, the population in this study was diverse, including patients with heart disease, hypertension, and medicated patients (Freed et al. 1994). Current HRV methodology guidelines (Task Force of the European Society of Cardiology and the North American Society of Pacing and Electrophysiology 1996) recommend that short-term measures of HRV be at least five-minutes in length to allow for accurate analysis of HRV variables. However, these guidelines do not indicate when this recording should be determined within a resting protocol. Rather the guidelines recommend only that the recordings be obtained during physiologically stable conditions (Task Force of the European Society of Cardiology and the North American Society of Pacing and Electrophysiology 1996). Consequently, this broad recommendation has resulted in a wide range of interpretations for "stable conditions" by researchers and numerous different protocols for resting HRV assessment (Leicht et al. 2003b; Sandercock et al. 2004; Tulppo et al. 1998; Yamamoto et al. 1991). This lack of consistency for resting "stable conditions" makes it impractical to compare the results of different 
studies and supports further the need for an examination of HRV short-term stability during rest.

Therefore, the current study aimed to assess the short-term stability and reproducibility of resting HRV during a 40-minute resting trial and the impact of body position and gender on these measures. Supine, seated, and standing resting positions were examined as these were the typical resting positions utilised for HRV examination, particularly for clinical investigations (Pomeranz et al. 1985; Sandercock et al. 2004). Gender was also examined as gender differences in HRV have been reported (Bigger et al. 1995; Van Hoogenhuyze et al. 1991) with the impact of gender on HRV stability unknown. Tenminute HRV recording lengths were examined in the current study due to their frequent use (Freed et al. 1994; Guijt et al. 2007; Marks and Lightfoot 1999; Sandercock et al. 2004) and in accordance with the current guidelines for HRV monitoring (Task Force of the European Society of Cardiology and the North American Society of Pacing and Electrophysiology 1996).

\section{Materials and Methods}

Participants

Thirty (14 males, 16 females) healthy adults volunteered to participate in this study with their descriptive characteristics shown in Table 1. Participants completed a general health pre-screening questionnaire and provided informed written consent prior to participation in accordance with the Declaration of Helsinki (2000) and approval by the local institutional Human Ethics Sub-committee.

\section{Design}

HRV data were assessed during 40-minute trials of three common resting positions (supine, seated, and standing rest) where the stability of resting HRV was assessed by comparing the data in consecutive 10-minute segments. Each resting position was examined on separate days and in a randomised order, following a 12-hour fast and a 24-hour abstinence from exercise. Participants completed all trials at a similar time of day to minimise the known circadian rhythm of HRV (Fallen and Kamath 1995) with an average of $2.2 \pm 0.9$ days separating each trial. All trials were completed in a controlled environment within a climate control chamber of mean $( \pm \mathrm{SD})$ temperature $24.6 \pm 0.5^{\circ} \mathrm{C}$, and mean relative humidity $52.8 \pm 2.7 \%$. As prior studies have reported equivocal results concerning the effect of the menstrual cycle on HRV (Leicht et al. 2003a; Sato et al. 1995), female participants in the current study completed their resting trials during the same phase of the menstrual cycle (days 16-28 after the first day of menses - luteal phase).

\section{Procedures}

Upon arrival at the laboratory, height was measured with a wall-mounted stadiometer (Seca, Hamburg, Germany), and mass was determined via bioelectrical impedance analysis (Tanita Corporation, Tokyo, Japan). Body mass index (BMI) was calculated as mass/height in metres squared. Three ECG monitoring electrodes (3M Australia Pty Ltd., Brisbane, Australia) were placed on prepared sites at the manubrium, left 5th intercostal space, and the right 5th/6th intercostal space for continuous ECG monitoring. Following preparation, participants were given a 1-2 minute customisation period before the 40-minute trial began. The single lead ECG (Lead II configuration) was sampled via a bioamplifier and Chart ${ }^{\circledR}$ software (v5.5; AD Instruments Pty Ltd., Bella Vista, Australia) at a sampling rate of 1 $\mathrm{kHz}$. Simultaneously with the ECG monitoring, respiratory gas analysis was conducted with expired gases being collected via a 2-way valve breathing mouthpiece (Hans Rudolph Inc., Kansas City, MO, USA) and sampled with an automated metabolic system (Powerlab 8M [AD Instruments Pty Ltd., Bella Vista, Australia] or Quinton [Quinton Instruments Co., Bothell, WA, USA]) calibrated according to the manufacturers guidelines. Respiratory rate was not controlled in the current study as previous research 
has reported similar reproducibility of HRV measures between spontaneous and controlled breathing (Hirsch and Bishop 1981; Zollei et al. 2007), and at various resting breathing frequencies (Patwardhan et al. 1995). All ECG and respiratory data were stored for later analysis.

\section{Data Analysis}

Respiratory parameters were derived from the automated systems as 15-second averages for the following variables: tidal volume (TV), respiratory rate $(R R)$, ventilation $\left(\dot{V}_{E}\right)$, and oxygen uptake $\left(\mathrm{V}_{2}\right)$. Respiration rate and depth were monitored during each resting position with all participants exhibiting a steady breathing pattern.

All ECG recordings were manually checked for missing and/or ectopic beats. Ectopic beats were excluded from the HRV analysis and were replaced via linear interpolation of the prior and following normal RR intervals. Each 10-minute segment of the 40-minute ECG recording was analysed using the HRV extension of Chart $^{\circledR}$ (v1.1; AD Instruments Pty Ltd., Bella Vista, Australia) for time-domain, frequency-domain and geometric measures of HRV (Table 2). The frequency-domain components of HRV were obtained via power spectral analysis of the data using a 1024-point FFT with an overlap of $50 \%$ for successive segments. A Hanning window was applied to each spectrum to reduce spectral leakage. All frequency domain components were expressed in absolute terms $\left(\mathrm{ms}^{2}\right)$ while LF and HF were also expressed in normalised units (nu) and as a ratio (Table 2) (Task Force of the European Society of Cardiology and the North American Society of Pacing and Electrophysiology 1996).

\section{Statistical analysis}

Data were analysed using the Statistical Package for the Social Sciences (SPSS v17, SPSS Inc., Chicago, ILL). Data normality was determined using the Kolmogorov-Smirnov statistic with a Lilliefors significance correction. All HRV measures [except HR, LF (nu) and HF (nu)] and TV exhibited a non-normal distribution and subsequently were transformed using a natural logarithm (ln) prior to statistical analyses. Significant differences between genders for descriptive characteristics were determined using independent t-tests or Mann-Whitney tests, where appropriate. Significant differences between variables were examined using 3-way (time $\mathrm{x}$ position $\mathrm{x}$ gender) repeated measures ANOVA and Tukey's post-hoc tests.

Reproducibility of HRV was determined via two statistical procedures: one-way random effects model ICC, as previously described (Zollei et al. 2007); and CV [CV=SD/mean] (Atkinson and Nevill 1998). Reproducibility was considered excellent for ICCs $>0.80$, and substantial when ICCs ranged between 0.60 and 0.80 (Pinna et al. 2007), while a CV $<10 \%$ was considered as acceptable (Atkinson and Nevill 1998) for HRV reproducibility. A p value $<0.05$ was set as the level of significance with all data expressed as mean \pm standard deviation.

\section{Results}

Despite a similar age, males were significantly taller and heaver resulting in a greater BMI compared to females (Table 1).

\section{Influence of time}

The main effect of time on HRV is summarised in Figure 1. Heart rate was stable until the final 10 minutes of the 40-minute trial period, when it increased significantly ( $\mathrm{p}=0.009$, Table 2 ). Of the timedomain and geometric variables, SD $\triangle N N$, RMSSD, and SD1 were constant throughout the entire resting period ( $p>0.05)$, while both SDNN and SD2 increased significantly during the final 20 minutes 
$(\mathrm{p}<0.001$, Table 3).

No significant effect of time was observed for the HF $\left(\mathrm{ms}^{2}\right)$ component, however all other frequencydomain variables exhibited significant changes over the 40-minute trial period $(\mathrm{p}<0.001$, Table 3 ). Compared to the first 10 minutes of rest, TP, LF $\left(\mathrm{ms}^{2}\right), \mathrm{LF}(\mathrm{nu})$ and LF/HF were significantly increased during the final 20 mins of rest (Table 3). VLF was significantly increased from 10-min onwards while $\mathrm{HF}$ (nu) was significantly lower after 20 minutes (Table 3). Unlike HRV, all respiratory measures were similar across the 40-minute resting period (Table 3). No significant interaction effects (i.e. time $\mathrm{x}$ gender, time $\mathrm{x}$ position $\mathrm{x}$ gender) were observed for any of the above HRV or respiratory variables.

\section{Influence of position}

Heart rate was significantly increased from supine through to standing rest with a corresponding significant decrease of all time-domain and geometric measures of HRV ( $<<0.001$, Table 4). A significant main effect for position was observed for all frequency-domain variables $(\mathrm{p}<0.001$, Table 4) with TP, LF (nu), HF $\left(\mathrm{ms}^{2}\right)$, HF (nu) and LF/HF measures significantly decreased from supine to seated rest, with a further significant decrease to standing rest. Similarly, VLF and LF $\left(\mathrm{ms}^{2}\right) \mathrm{were}^{2}$ significantly reduced during standing rest, compared to supine and seated rest (Table 4). No significant interaction effects (i.e. position $\mathrm{x}$ time, position $\mathrm{x}$ gender, position $\mathrm{x}$ time $\mathrm{x}$ gender) were observed for any of the HRV variables except for a significant position $x$ time interaction effect for VLF ( $p=0.041$, Table 5). This interaction reflected a significantly lower VLF during standing compared to supine at each 10-minute period and greater VLF during the final 20 minutes of supine and seated rest (Table 5).

Although TV was similar during all resting positions, RR, $\dot{\mathrm{V}}_{\mathrm{E}}$, and $\dot{\mathrm{V}} \mathrm{O}_{2}$ were significantly increased from supine and seated rest, to standing rest $(\mathrm{p}<0.001$; Table 4). No significant interaction effects (i.e. position $\mathrm{x}$ time, position $\mathrm{x}$ gender, position $\mathrm{x}$ time $\mathrm{x}$ gender) were observed for any of the HRV variables except for a significant position $x$ time interaction effect for $\dot{\mathrm{V}}_{\mathrm{E}}$ and $\dot{\mathrm{V}} \mathrm{O}_{2}(\mathrm{p}=0.009$ and $\mathrm{p}=$ 0.036, Table 5). Standing resulted in a significantly greater $\dot{\mathrm{V}}_{\mathrm{E}}$ and $\dot{\mathrm{V}} \mathrm{O}_{2}$ compared to supine and seated rest at each 10-minute period while significantly lower $\dot{\mathrm{V}}_{\mathrm{E}}$ was observed during the final 20 minutes of supine rest (Table 5).

\section{Influence of gender}

Compared to females, males exhibited significantly greater SDNN, SD2, TP, VLF, LF (ms ${ }^{2}$ ), LF(nu), LF/HF and lower ( $\mathrm{p}<0.06) \mathrm{HR}$ and HF (nu) (Table 6). Similarly, males exhibited greater TV, $\dot{\mathrm{V}}_{\mathrm{E}}$ and $\dot{\mathrm{V}} \mathrm{O}_{2}$ compared to females (Table 6). No significant interaction effects (i.e. gender $\mathrm{x}$ time, gender $\mathrm{x}$ position, gender $\mathrm{x}$ time $\mathrm{x}$ position) were observed for any of the variables.

\section{Reproducibility}

Most HRV and all respiratory variables demonstrated substantial to excellent reproducibility as assessed by ICC $(\mathrm{p}<0.001)$ for all resting positions and genders (Table 7). Similarly, the CV for HR and most respiratory variables exhibited acceptable reproducibility (i.e. $\leq 10 \%$ ) for all resting positions and genders (Table 7). In contrast, the $\mathrm{CV}$ s for the time-domain and geometric variables were greater and ranged between 8.9 to $23.8 \%$. Similarly, the $\mathrm{CV}$ for the frequency domain variables were large and ranged between 7.1 and 53.6\% (Table 7). Of particular note was that the LF (nu) and HF (nu) variables exhibited lower CVs than the corresponding absolute measures of LF and HF ( $\left.\mathrm{ms}^{2}\right)$ (Table 7).

\section{Discussion}

The results of this study demonstrated that time-domain, geometric, and frequency domain measures of 
HRV fluctuate over time and therefore were not stable during supine, seated, or standing rest. Significant position and gender effects were observed with greater HRV exhibited during supine rest and for males. The reproducibility of the examined HRV variables was generally substantial (ICCs >0.6) with the time-domain and geometric measures of HRV demonstrating greater reproducibility (higher ICCs and lower CVs) compared to the frequency domain parameters. The lack of HRV stability during rest in the current study raises concerns about current resting methodologies and confirms the need for a standardised resting HRV protocol to be used in conjunction with the current HRV guidelines (Task Force of the European Society of Cardiology and the North American Society of Pacing and Electrophysiology 1996) for comparison of HRV between studies.

As demonstrated in Figure 1, HRV parameters were variable throughout the 40-minute resting period. The HRV measures reported to reflect vagal modulations of HR [i.e. SD $\Delta$ NN, RMSSD, SD1, and HF $\left(\mathrm{ms}^{2}\right)$ ] (Task Force of the European Society of Cardiology and the North American Society of Pacing and Electrophysiology 1996) were similar throughout the 40-minute rest recording while variables influenced by sympathetic and/or other physiological modulations such as SDNN, TP, LF (ms ${ }^{2}$ ), LF (nu) and LF/HF (Task Force of the European Society of Cardiology and the North American Society of Pacing and Electrophysiology 1996) were significantly increased 20-minutes into the recording. The frequency-domain measures of HRV provide a key insight into the physiological and neural influence of HR control, particularly within short-term recordings (Task Force of the European Society of Cardiology and the North American Society of Pacing and Electrophysiology 1996). Although stability in the HF component suggests that vagal influence on HR was unchanged over the recording period, TP which reflects primarily vagal modulations (Task Force of the European Society of Cardiology and the North American Society of Pacing and Electrophysiology 1996) was significantly increased, most likely due to an increased LF power and a larger increase in VLF power. Since the LF component has been suggested as an indicator of vagal and sympathetic modulation of HR (Akselrod et al. 1981; Akselrod et al. 1985; Pomeranz et al. 1985), it is reasonable to conclude that sympathetic activity may have increased over the 40-minute trial period. In a study examining the effect of mental stress on HRV, it was cautioned that an increased LF component may not be reflective of sympathetic activity, but by alterations in breathing frequencies associated with various tasks (Bernardi et al. 2000). However, this was unlikely in the current study as RR remained stable over the whole 40-minute period (Table 3). Further, the reproducibility of these respiratory parameters was observed to be excellent (ICCs >0.85; CVs ranging between 5.3 and 11.0\%). Additionally, changes in arterial pressure with enhanced baroreflex activity are also known to affect the LF component of HRV (Akselrod et al. 1981) and may have impacted on HRV in the current study however, blood pressure was not monitored and this remains to be examined in future studies.

More difficult to explain was the observed increase in the long-term HRV measures (e.g. VLF). Since vagal influence was demonstrated to remain stable (i.e. HF power was unchanged over time), other factors may contribute significantly to the increased VLF. It seems unlikely that the increase in VLF was a result of thermoregulatory system fluctuations (Kitney 1977) since all trials were completed in a constant thermoneutral environment. Similarly, the steady environment and the inclusion of only healthy participants into the study suggest that the increased VLF was not a result of a change in peripheral chemoreceptor sensitivity (Ponikowski et al. 1996). Possible changes in renin-angiotensin system (RAS) activity (Akselrod et al. 1981; Akselrod et al. 1985) and/or parasympathetic activity at very low frequencies (Taylor et al. 1998) may have contributed to the enhanced VLF. Greater VLF during the last 20 mins of supine and seated rest, to a level greater than that evident during standing (Table 4), may suggest reduced RAS in response to reduced baroreflex activity (Akselrod et al. 1981; Akselrod et al. 1985) during supine and seated rest. Subsequently, reduced RAS activity may attenuate the inhibitory effect of Angiotensin II on the sino-atrial node increasing the very low vagal oscillations 
and HRV (i.e. VLF). However, the influence of RAS activity and blood pressure changes remains to be confirmed as blood pressure was not monitored in the current study. Future examination of simultaneous HR and BP may give further insight into the role of the VLF component and its physiological mechanisms in regulating HRV stability during rest.

The aims of the current study were to examine the short-term stability of resting HRV and the impact of body position and gender on HRV stability. While HRV fluctuated over time, HRV magnitude rather than reproducibility was influenced by position and gender. In line with previous reports, HRV was significantly decreased from supine to seated rest, and further decreased during standing rest (Pomeranz et al. 1985). Despite the absolute reduction in HRV with resting position, reproducibility as assessed by ICC and CV was similar for each position (Table 7) and suggests that HRV can be consistently assessed during different resting positions, albeit the same resting position. Similarly, HRV reproducibility was comparable for males and females (Table 7) despite significantly greater HRV for males (Table 6). Evidence from studies suggesting HRV differences between genders has been debatable with several studies reporting no significant differences in HRV between male and female healthy subjects (Sinnreich et al. 1998). Others have observed a significantly greater HRV in healthy male subjects (Bigger et al. 1995; Van Hoogenhuyze et al. 1991). Lower HRV for females has been suggested to possibly reflect gender comparisons at different menstrual cycle stages (Leicht et al. 2003a) with greater sympathetic activity and lower parasympathetic activity reported during the luteal phase compared with the follicular stage (Saeki et al. 1997; Sato et al. 1995). However, this seems unlikely in the current study as HRV was reported to be similar at times of high and low ovarian hormonal influence in young healthy females (Leicht et al. 2003a). Regardless, the similar HRV stability for males and females, suggests that HRV can be consistently assessed for males and females, albeit separately.

The reproducibility of HR in the current study was excellent (ICCs>0.9, CVs <4\%, Table 7) during all resting positions for males and females. Similarly, most HRV variables demonstrated substantial reproducibility for all resting positions in terms of ICCs (>0.6). Kowalewski and Urban (2004) obtained multiple (though not adjacent) 10-minute recordings of HRV during same-day recordings and reported ICCs ranging from 0.54-0.89 in the supine positions, and 0.68-0.91 in the standing positions for TP, LF, HF, and the LF/HF. Likewise, the current study demonstrated similar ICCs ranging from 0.721-0.931 for these variables in the supine position and 0.546-0.933 in the standing position. However, the current study's CV was rather large, particularly for the frequency-domain measures (Table 7). Notably, those measures of HRV that reflected vagal modulation of HR generally exhibited better ICCs than the other variables (e.g. 0.820-0.956 during supine rest for SD $\triangle N N$, RMSSD, SD1, and HF; compared to 0.699-0.865 for all other variables during supine rest). Further, the CVs of time and frequency-domain variables in the current study were greater than that reported for patients with CAD, MI and hypertension (e.g. 20-41\% vs. 9-15\%) (Freed et al. 1994; Tarkiainen et al. 2005). The healthy population utilised in the current study may account for the differences in CVs as these young, healthy, active participants exhibited greater HRV and potentially greater variability of HR and HRV (both within- and between-participants). Similar large CVs in healthy children with high HRV and developing neural systems (Leicht and Allen 2008) further support the notion that large CVs for HRV may in fact be typical in healthy populations with high HRV. In contrast, lower CVs exhibited by patients with cardiovascular disease (Freed et al. 1994; Tarkiainen et al. 2005) may be a result of low HRV and a more dominant sympathetic influence commonly reported for these patients (Camm et al. 2004).

\section{Limitations}

It should be noted that the current results were limited to healthy, young, active adults. To our 
knowledge, short-term stability of HRV and the requirement for a standardised resting protocol for other populations, including youths, elderly and those suffering from cardiological or non-cardiological disease, remains to be confirmed.

\section{Recommended standardised protocol}

Within the current study, HRV did not remain consistent during rest and makes it difficult to compare results from different studies utilising different resting protocols. Subsequently, a standardised resting protocol may be beneficial to allow direct comparison between studies. Based on the current results, we would suggest that HRV be assessed at 0-10 minutes of rest. Although most variables were stable during the final 10 minutes of the recording, this period was deemed unsuitable because of a significant increase in HR. Similarly, the 10-20 and 20-30 minute segments were excluded due to increases of the VLF and/or other HRV measures (Table 3, Figure 1). For the purposes of clinical evaluation, the 0-10 minute segment provides the most time efficient option however, it could be debated as to whether this segment is truly reflective of a resting state. Gujit and colleagues (2007) compared 10 minutes of supine rest (following a five-minute acclimatisation period) to the second hour of an ambulatory night recording and found significant differences between the two. Their recommendation was that caution needed to be used within the laboratory, as a laboratory-based protocol may not necessarily reflect a true resting state.

In the current study, most HRV variables had reached peak values by the 20-30 minute period that may indicate this timeframe as the most suitable for the recording of HRV and a similar resting state to that of night-time recordings, particularly in terms of the VLF component. However, this timeframe should be considered cautiously as $\mathrm{HF}(\mathrm{nu})$ and LF/HF were also significantly altered indicating possibly enhanced sympathetic modulations (Task Force of the European Society of Cardiology and the North American Society of Pacing and Electrophysiology 1996). Identification of the physiological mechanisms responsible for the increased HRV during the 20-30 minute segment of the current study is particularly important since VLF has been suggested as a powerful predictor of cardiac events (Hadase et al. 2004). Further research examining HRV with the assessment of blood pressure may assist in determining whether blood pressure variability influences the observed increases in LF and VLF, and may clarifying if the 20-30 minute timeframe is an appropriate period for 'stable resting HRV' measurements.

The current study determined that some short-term HRV parameters do not remain constant during normal resting conditions although they exhibit substantial reproducibility for supine, seated and standing positions for males and females. It was recommended that HRV (10-minute recording) be assessed during 0-10 minutes in any standard resting position (i.e. supine, seated, standing) for both males and females. Use of this standardised resting protocol will enable comparison of short-term HRV between studies as a valid research and clinical tool.

\section{Acknowledgements}

The authors would like to thank Jason Greenway and Sarah Conn for assistance with data collection. 


\section{References}

Akselrod, S., Gordon, D., Ubel, F.A., Shannon, D.C., Berger, A.C., and Cohen, R.J. 1981. Power spectrum analysis of heart rate fluctuation: a quantitative probe of beat-to-beat cardiovascular control. Science. 213(4504): 220-222.

Akselrod, S., Gordon, D., Madwed, J.B., Snidman, N.C., Shannon, D.C., and Cohen, R.J. 1985. Hemodynamic regulation: investigation by spectral analysis. Am. J. Physiol. 249(4 Pt 2): H867875.

Atkinson, G. and Nevill, A.M. 1998. Statistical methods for assessing measurement error (reliability) in variables relevant to sports medicine. Sports Med. 26(4): 217-238.

Aubert, A.E., Beckers, F., and Ramaekers, D. 2001. Short-term heart rate variability in young athletes. J. Cardiol. 37(Suppl 1): 85-88.

Bernardi, L., Wdowczyk-Szulc, J., Valenti, C., Castoldi, S., Passino, C., Spadacini, G., and Sleight, P. 2000. Effects of controlled breathing, mental activity and mental stress with or without verbalization on heart rate variability. J. Am. Coll. Cardiol. 35(6): 1462-1469.

Bigger, J.T., Jr., Fleiss, J.L., Steinman, R.C., Rolnitzky, L.M., Schneider, W.J., and Stein, P.K. 1995. RR variability in healthy, middle-aged persons compared with patients with chronic coronary heart disease or recent acute myocardial infarction. Circulation. 91(7): 1936-1943.

Camm, A.J., Pratt, C.M., Schwartz, P.J., Al-Khalidi, H.R., Spyt, M.J., Holroyde, M.J., Karam, R., Sonnenblick, E.H., and Brum, J.M. 2004. Mortality in patients after a recent myocardial infarction: a randomized, placebo-controlled trial of Azimilide using heart rate variability for risk stratification. Circulation. 109(8): 990-996.

Fallen, E.L. and Kamath, M.V. 1995. Circadian rhythms of heart rate variability. In Heart rate variability. Edited by M. Malik and A.J. Camm. Futura Publishing Company Inc., Armonk, NY. pp. 293-310.

Fei, L., Copie, X., Malik, M., and Camm, A.J. 1996. Short- and long-term assessment of heart rate variability for risk stratification after acute myocardial infarction. Am. J. Cardiol. 77(9): 681684.

Freed, L.A., Stein, K.M., Gordon, M., Urban, M., and Kligfield, P. 1994. Reproducibility of power spectral measures of heart rate variability obtained from short-term sampling periods. Am. J. Cardiol. 74(9): 972-973.

Guijt, A.M., Sluiter, J.K., and Frings-Dresen, M.H.W. 2007. Test-retest reliability of heart rate variability and respiration rate at rest and during light physical activity in normal subjects. Arch. Med. Res. 38(1): 113-120.

Hadase, M., Azuma, A., Zen, K., Asada, S., Kawasaki, T., Kamitani, T., Kawasaki, S., Sugihara, H., and Matsubara, H. 2004. Very low frequency power of heart rate variability is a powerful predictor of clinical prognosis in patients with congestive heart failure. Circ J. 68(4): 343-347.

Hirsch, J.A. and Bishop, B. 1981. Respiratory sinus arrhythmia in humans: how breathing pattern modulates heart rate. Am. J. Physiol. 241(4): H620-629.

Kitney, R.I. 1977. Magnitude and phase changes in heart rate variability and blood pressure during respiratory entrainment [proceedings]. J Physiol. 270(1): 40P-41P.

Kleiger, R.E., Miller, J.P., Bigger, J.T., Jr., and Moss, A.J. 1987. Decreased heart rate variability and its association with increased mortality after acute myocardial infarction. Am. J. Cardiol. 59(4): 256-262.

Kowalewski, M.A. and Urban, M. 2004. Short- and long-term reproducibility of autonomic measures in supine and standing positions. Clin. Sci. (London). 106(1): 61-66.

Leicht, A.S. and Allen, G.D. 2008. Moderate-term reproducibility of heart rate variability during rest and light to moderate exercise in children. Braz. J. Med. Biol. Res. 41(7): 627-633.

Leicht, A.S., Hirning, D.A., and Allen, G.D. 2003a. Heart rate variability and endogenous sex hormones during the menstrual cycle in young women. Exp. Physiol. 88(3): 441-446. 
Leicht, A.S., Allen, G.D., and Hoey, A.J. 2003b. Influence of intensive cycling training on heart rate variability during rest and exercise. Can. J. Appl. Physiol. 28(6): 898-909.

Marks, B.L. and Lightfoot, J.T. 1999. Reproducibility of resting heart rate variability with short sampling periods. Can. J. Appl. Physiol. 24(4): 337-348.

Patwardhan, A.R., Evans, J.M., Bruce, E.N., Eckberg, D.L., and Knapp, C.F. 1995. Voluntary control of breathing does not alter vagal modulation of heart rate. J. Appl. Physiol. 78(6): 2087-2094.

Pinna, G.D., Maestri, R., Torunski, A., Danilowicz-Szymanowicz, L., Szwoch, M., La Rovere, M.T., and Raczak, G. 2007. Heart rate variability measures: a fresh look at reliability. Clin. Sci. (London). 113(3): 131-140.

Pomeranz, B., Macaulay, R.J., Caudill, M.A., Kutz, I., Adam, D., Gordon, D., Kilborn, K.M., Barger, A.C., Shannon, D.C., Cohen, R.J., and Benson, H. 1985. Assessment of autonomic function in humans by heart rate spectral analysis. Am. J. Physiol. 248(1 Pt 2): H151-153.

Ponikowski, P., Chua, T.P., Amadi, A.A., Piepoli, M., Harrington, D., Volterrani, M., Colombo, R., Mazzuero, G., Giordano, A., and Coats, A.J. 1996. Detection and significance of a discrete very low frequency rhythm in RR interval variability in chronic congestive heart failure. Am. J. Cardiol. 77(15): 1320-1326.

Saeki, Y., Atogami, F., Takahashi, K., and Yoshizawa, T. 1997. Reflex control of autonomic function induced by posture change during the menstrual cycle. J. Auton. Nerv. Syst. 66(1-2): 69-74.

Sandercock, G.R., Bromley, P., and Brodie, D.A. 2004. Reliability of three commercially available heart rate variability instruments using short-term (5-min) recordings. Clin. Physiol. Funct. Imaging. 24(6): 359-367.

Sandercock, G.R., Bromley, P.D., and Brodie, D.A. 2005. The reliability of short-term measurements of heart rate variability. Int. J. Cardiol. 103(3): 238-247.

Sato, N., Miyake, S., Akatsu, J., and Kumashiro, M. 1995. Power spectral analysis of heart rate variability in healthy young women during the normal menstrual cycle. Psychosom. Med. 57(4): 331-335.

Sinnreich, R., Kark, J.D., Friedlander, Y., Sapoznikov, D., and Luria, M.H. 1998. Five minute recordings of heart rate variability for population studies: repeatability and age-sex characteristics. Heart. 80(2): 156-162.

Tarkiainen, T.H., Timonen, K.L., Tiittanen, P., Hartikainen, J.E., Pekkanen, J., Hoek, G., Ibald-Mulli, A., and Vanninen, E.J. 2005. Stability over time of short-term heart rate variability. Clin. Auton. Res. 15(6): 394-399.

Task Force of the European Society of Cardiology and the North American Society of Pacing and Electrophysiology. 1996. Heart rate variability: Standards of measurement, physiological interpretation and clinical use. Circulation. 93(5): 1043-1065.

Taylor, J.A., Carr, D.L., Myers, C.W., and Eckberg, D.L. 1998. Mechanisms underlying very-lowfrequency RR-interval oscillations in humans. Circulation. 98(6): 547-555.

Tsuji, H., Venditti, F.J., Jr., Manders, E.S., Evans, J.C., Larson, M.G., Feldman, C.L., and Levy, D. 1994. Reduced heart rate variability and mortality risk in an elderly cohort. The Framingham Heart Study. Circulation. 90(2): 878-883.

Tulppo, M.P., Makikallio, T.H., Seppanen, T., Laukkanen, R.T., and Huikuri, H.V. 1998. Vagal modulation of heart rate during exercise: effects of age and physical fitness. Am. J. Physiol. 274(2 Pt 2): H424-429.

Van Hoogenhuyze, D., Weinstein, N., Martin, G.J., Weiss, J.S., Schaad, J.W., Sahyouni, X.N., Fintel, D., Remme, W.J., and Singer, D.H. 1991. Reproducibility and relation to mean heart rate of heart rate variability in normal subjects and in patients with congestive heart failure secondary to coronary artery disease. Am. J. Cardiol. 68(17): 1668-1676.

Yamamoto, Y., Hughson, R.L., and Peterson, J.C. 1991. Autonomic control of heart rate during exercise studied by heart rate variability spectral analysis. J. Appl. Physiol. 71(3): 1136-1142. 
Zollei, E., Csillik, A., Rabi, S., Gingl, Z., and Rudas, L. 2007. Respiratory effects on the reproducibility of cardiovascular autonomic parameters. Clin. Physiol. Funct. Imaging. 27(4): 205-210. 
Table 1 Participant characteristics.

\begin{tabular}{lccc}
\hline & $\begin{array}{c}\text { Male } \\
(\mathrm{n}=14)\end{array}$ & $\begin{array}{c}\text { Female } \\
(\mathrm{n}=16)\end{array}$ & $\begin{array}{c}\text { All } \\
(\mathrm{n}=30)\end{array}$ \\
\hline Age $(\mathrm{yr})$ & $24.2 \pm 5.9$ & $24.9 \pm 7.8$ & $24.6 \pm 6.9$ \\
Height $(\mathrm{cm})$ & $180.9 \pm 6.8$ & $165.9 \pm 8.3 * * *$ & $172.9 \pm 10.7$ \\
Mass $(\mathrm{kg})$ & $79.8 \pm 10.9$ & $59.4 \pm 8.8^{* * *}$ & $68.9 \pm 14.2$ \\
BMI $\left(\mathrm{kg} \cdot \mathrm{m}^{-2}\right)$ & $24.3 \pm 2.8$ & $21.5 \pm 2.1 * *$ & $22.8 \pm 2.8$ \\
\hline$* * \mathrm{p}<0.01, * * * \mathrm{p}<0.001$ vs. Male & &
\end{tabular}


Table 2 Time and frequency domain measures of heart rate variability assessed in the current study.

\begin{tabular}{ll}
\hline Measure & \multicolumn{1}{c}{ Description* } \\
\hline SDNN & Standard deviation of all normal-normal RR intervals \\
SD $\Delta$ NN & Standard deviation of the change in all normal-normal RR intervals \\
RMSSD & The root mean square of successive differences in normal-normal RR intervals \\
SD1 & Standard deviation of instantaneous RR variability (i.e. short axis of Poincare plot) \\
SD2 & Standard deviation of long-term RR variability (i.e. long axis of the Poincare plot, \\
VLF & Spectral power located within the very low frequency range (0-0.04 Hz) and represents \\
& modulations of the parasympathetic and sympathetic nervous systems, the renin- \\
& angiotensin system, the thermoregulatory system, and chemoreflex sensitivity (Akselrod \\
& et al. 1985; Ponikowski et al. 1996) \\
LF & Spectral power located within the low frequency range (0.04-0.15 Hz) and reflects \\
& modulations of the parasympathetic and sympathetic nervous systems including \\
& baroreflex activity (Akselrod et al. 1981) \\
HF & Spectral power located within the high frequency range (0.15-0.4 Hz) and predominately \\
& reflects modulations of the parasympathetic nervous system (Akselrod et al. 1981) \\
TP & Spectral power located within the total frequency range (0-0.4 Hz) \\
LF/HF & Ratio of LF to HF and indicator of sympathovagal balance \\
\hline
\end{tabular}

*(Task Force of the European Society of Cardiology and the North American Society of Pacing and Electrophysiology 1996) 
Table 3 Heart rate variability and respiratory variables during consecutive 10-minute resting periods $(\mathrm{n}=30)$.

\begin{tabular}{|c|c|c|c|c|}
\hline & \multicolumn{4}{|c|}{ Time (minutes) } \\
\hline & 0-10 & $10-20$ & $20-30$ & $30-40$ \\
\hline HR (bpm) & $68.3 \pm 15.5$ & $69.0 \pm 15.7$ & $69.2 \pm 15.6$ & $70.0 \pm 16.5^{*}$ \\
\hline SDNN (ms) & $77.6 \pm 32.3$ & $82.0 \pm 36.8$ & $87.6 \pm 36.8^{* *}$ & $88.5 \pm 38.3^{* *}$ \\
\hline $\mathrm{SD} \Delta \mathrm{NN}(\mathrm{ms})$ & $66.4 \pm 47.7$ & $66.6 \pm 49.2$ & $67.6 \pm 47.0$ & $64.9 \pm 43.2$ \\
\hline RMSSD (ms) & $66.4 \pm 47.6$ & $66.5 \pm 49.1$ & $68.2 \pm 46.1$ & $64.9 \pm 43.1$ \\
\hline SD1 (ms) & $47.0 \pm 33.7$ & $47.1 \pm 34.8$ & $47.8 \pm 33.2$ & $45.9 \pm 30.5$ \\
\hline $\mathrm{SD} 2(\mathrm{~ms})$ & $97.7 \pm 35.5$ & $104.6 \pm 42.4$ & $112.9 \pm 43.7 * *$ & $115.4 \pm 47.5^{* * \dagger \dagger}$ \\
\hline $\mathrm{TP}\left(\mathrm{ms}^{2}\right)$ & $6661 \pm 6238$ & $8049 \pm 8181$ & $8826 \pm 7756^{* *}$ & $8673 \pm 7768^{* *}$ \\
\hline $\operatorname{VLF}\left(\mathrm{ms}^{2}\right)$ & $1847 \pm 1393$ & $2818 \pm 3037 * *$ & $3558 \pm 3334^{* * \dagger}$ & $3436 \pm 3531^{* *}$ \\
\hline $\mathrm{LF}\left(\mathrm{ms}^{2}\right)$ & $2038 \pm 2063$ & $2393 \pm 2661$ & $2585 \pm 2504 * *$ & $2731 \pm 2744 * *$ \\
\hline $\mathrm{LF}(\mathrm{nu})$ & $52.29 \pm 22.74$ & $54.81 \pm 21.59$ & $57.5 \pm 20.95^{* *}$ & $58.25 \pm 21.10^{* *}$ \\
\hline $\mathrm{HF}\left(\mathrm{ms}^{2}\right)$ & $2654 \pm 4455$ & $2586 \pm 4984$ & $2528 \pm 4130$ & $2362 \pm 3229$ \\
\hline $\mathrm{HF}(\mathrm{nu})$ & $44.75 \pm 22.36$ & $41.92 \pm 21.17$ & $39.49 \pm 20.79 * *$ & $38.79 \pm 20.74^{* *}$ \\
\hline $\mathrm{LF} / \mathrm{HF}$ & $2.42 \pm 3.63$ & $2.58 \pm 3.71$ & $2.76 \pm 3.64 * *$ & $3.03 \pm 4.05^{* *}$ \\
\hline TV (L) & $0.69 \pm 0.34$ & $0.69 \pm 0.35$ & $0.69 \pm 0.35$ & $0.70 \pm 0.34$ \\
\hline RR (breaths $\cdot \min ^{-1}$ ) & $14.4 \pm 4.0$ & $14.4 \pm 4.0$ & $14.4 \pm 3.7$ & $14.0 \pm 3.6$ \\
\hline$\dot{\mathrm{V}}_{\mathrm{E}}\left(\mathrm{L} \cdot \mathrm{min}^{-1}\right)$ & $7.78 \pm 2.33$ & $7.64 \pm 2.54$ & $7.61 \pm 2.60$ & $7.60 \pm 2.51$ \\
\hline$\dot{\mathrm{V}} \mathrm{O}_{2}\left(\mathrm{~mL} \cdot \mathrm{kg}^{-1} \cdot \mathrm{min}^{-1}\right)$ & $4.20 \pm 1.03$ & $4.15 \pm 1.19$ & $4.17 \pm 1.28$ & $4.22 \pm 1.28$ \\
\hline
\end{tabular}

Values are mean $\pm \mathrm{SD} ;{ }^{*} \mathrm{p}<0.05, * * \mathrm{p}<0.01$ vs. $0-10 ;{ }^{\dagger} \mathrm{p}<0.05,{ }^{\dagger \dagger} \mathrm{p}<0.01$ vs. 10-20; HR, heart rate; SDNN, standard deviation of RR (NN) intervals; SD $\triangle N N$, standard deviation of the change in RR intervals; RMSSD, square root of the mean squared differences of successive RR intervals; SD1, standard deviation of instantaneous RR variability; SD2, standard deviation of long-term RR variability; TP, total power; VLF, very low frequency; LF, low frequency; nu, normalised units; HF, high frequency; LF/HF, low frequency to high frequency ratio; $\mathrm{TV}$, tidal volume; $\mathrm{RR}$, respiratory rate; $\dot{\mathrm{V}}_{\mathrm{E}}$, ventilation; $\dot{\mathrm{V}} \mathrm{O}_{2}$, oxygen uptake. 
Table 4 Heart rate variability and respiratory variables during supine, seated, and standing resting positions $(n=30)$.

\begin{tabular}{|c|c|c|c|}
\hline & Supine & Seated & Standing \\
\hline HR (bpm) & $58.0 \pm 9.8$ & $67.0 \pm 10.6^{* *}$ & $82.3 \pm 15.5^{* * \dagger \dagger}$ \\
\hline SDNN (ms) & $105.9 \pm 37.6$ & $85.4 \pm 30.1 * *$ & $60.4 \pm 24.4 * * \dagger$ \\
\hline $\mathrm{SD} \Delta \mathrm{NN}(\mathrm{ms})$ & $103.9 \pm 51.6$ & $62.7 \pm 28.9 * *$ & $32.5 \pm 21.5^{* * \dagger \dagger}$ \\
\hline RMSSD (ms) & $103.6 \pm 51.4$ & $62.7 \pm 28.9^{* *}$ & $33.2 \pm 22.1 * * \dagger$ \\
\hline $\mathrm{SD} 1(\mathrm{~ms})$ & $73.5 \pm 36.5$ & $44.4 \pm 20.4 * *$ & $23.0 \pm 15.2 * * \dagger \dagger$ \\
\hline $\mathrm{SD} 2$ (ms) & $129.0 \pm 43.6$ & $111.9 \pm 38.9^{*}$ & $82.0 \pm 31.6 * * \dagger$ \\
\hline $\mathrm{TP}\left(\mathrm{ms}^{2}\right)$ & $12251 \pm 9277$ & $7782 \pm 5963 * *$ & $4123 \pm 4057 * * \dagger$ \\
\hline $\operatorname{VLF}\left(\mathrm{ms}^{2}\right)$ & $3658 \pm 3516$ & $3171 \pm 2918$ & $1915 \pm 2206 * * \dagger \dagger$ \\
\hline $\mathrm{LF}\left(\mathrm{ms}^{2}\right)$ & $3197 \pm 2798$ & $2584 \pm 2861$ & $1529 \pm 1247^{* * \dagger}$ \\
\hline $\mathrm{LF}(\mathrm{nu})$ & $40.86 \pm 19.35$ & $53.48 \pm 18.30^{* *}$ & $72.80 \pm 13.50 * * \dagger$ \\
\hline $\mathrm{HF}\left(\mathrm{ms}^{2}\right)$ & $5073 \pm 6287$ & $1904 \pm 1779 * *$ & $621 \pm 858 * *+\dagger$ \\
\hline $\mathrm{HF}(\mathrm{nu})$ & $55.98 \pm 18.93$ & $43.26 \pm 18.15^{* *}$ & $24.47 \pm 13.27 * * \dagger \dagger$ \\
\hline $\mathrm{LF} / \mathrm{HF}$ & $1.15 \pm 1.51$ & $2.18 \pm 3.61^{* *}$ & $4.77 \pm 4.49 * * \dagger \dagger$ \\
\hline $\mathrm{TV}(\mathrm{L})$ & $0.64 \pm 0.27$ & $0.67 \pm 0.36$ & $0.76 \pm 0.38$ \\
\hline RR (breaths $\cdot \min ^{-1}$ ) & $13.58 \pm 3.77$ & $14.04 \pm 3.66$ & $15.26 \pm 3.83 * * \dagger$ \\
\hline$\dot{\mathrm{V}}_{\mathrm{E}}\left(\mathrm{L} \cdot \mathrm{min}^{-1}\right)$ & $6.78 \pm 1.78$ & $7.27 \pm 2.28$ & $8.92 \pm 2.79 * * \dagger \dagger$ \\
\hline$\dot{\mathrm{V}} \mathrm{O}_{2}\left(\mathrm{~mL} \cdot \mathrm{kg}^{-1} \cdot \mathrm{min}^{-1}\right)$ & $3.96 \pm 0.87$ & $3.86 \pm 1.12$ & $4.73 \pm 1.35^{* * \dagger \dagger}$ \\
\hline
\end{tabular}

Values are mean \pm SD; $* p<0.05, * * p<0.01$ vs. Supine; ${ }^{\dagger} p<0.05,{ }^{\dagger \dagger} p<0.01$ vs. Seated; HR, heart rate; SDNN, standard deviation of RR (NN) intervals; SD $\triangle N N$, standard deviation of the change in RR intervals; RMSSD, square root of the mean squared differences of successive RR intervals; SD1, standard deviation of instantaneous RR variability; SD2, standard deviation of long-term RR variability; TP, total power; VLF, very low frequency; $\mathrm{LF}$, low frequency; nu, normalised units; HF, high frequency; LF/HF, low frequency to high frequency ratio; $\mathrm{TV}$, tidal volume; $\mathrm{RR}$, respiratory rate; $\dot{\mathrm{V}}_{\mathrm{E}}$, ventilation; $\dot{\mathrm{V}} \mathrm{O}_{2}$, oxygen uptake. 
Table 5 Heart rate variability and respiratory variables during consecutive 10-minute periods of supine, seated and standing rest $(\mathrm{n}=30)$.

Time (minutes)
0-10
10-20
20-30
30-40

$\operatorname{VLF}\left(\mathrm{ms}^{2}\right)$

$\begin{array}{rcccc}\text { Supine } & 2014 \pm 1169 & 3296 \pm 3085 & 4598 \pm 3708^{\dagger \dagger} & 4725 \pm 4591^{\dagger \dagger} \\ \text { Seated } & 2083 \pm 1623 & 3077 \pm 3270 & 4013 \pm 3331^{\dagger \dagger} & 3510 \pm 2903^{\dagger \dagger} \\ \text { Standing } & 1445 \pm 1304 * * & 2080 \pm 2688 * *+\dagger & 2063 \pm 2370 * *+\dagger & 2071 \pm 2261^{* * \dagger+}\end{array}$

$\dot{\mathrm{V}}_{\mathrm{E}}\left(\mathrm{L} \cdot \mathrm{min}^{-1}\right)$

\begin{tabular}{|c|c|c|c|c|}
\hline Supine & $7.23 \pm 1.98$ & $6.77 \pm 1.82$ & $6.56 \pm 1.70^{\dagger}$ & $6.57 \pm 1.62^{\dagger}$ \\
\hline Seated & $7.30 \pm 2.09$ & $7.26 \pm 2.38$ & $7.28 \pm 2.42 * *$ & $7.23 \pm 2.35 * *$ \\
\hline tanding & $8.81 \pm 2.59 * * \$$ & $8.89 \pm 2.89 * * \hbar$ & $8.98 \pm 2.96^{* *+4}$ & $8.99 \pm 2.83 * * t$ \\
\hline
\end{tabular}

$\dot{\mathrm{V}} \mathrm{O}_{2}\left(\mathrm{ml} \cdot \mathrm{kg}^{-1} \cdot \mathrm{min}^{-1}\right)$

$\begin{array}{rcccc}\text { Supine } & 4.11 \pm 0.83 & 3.93 \pm 0.83 & 3.89 \pm 0.92 & 3.94 \pm 0.93 \\ \text { Seated } & 3.89 \pm 0.97 & 3.81 \pm 1.12 & 3.81 \pm 1.16 & 3.90 \pm 1.24 \\ \text { Standing } & 4.61 \pm 1.15^{* * * t} & 4.71 \pm 1.37 * * * t & 4.80 \pm 1.48 * * * & 4.81 \pm 1.44 * * *\end{array}$

Values are mean $\pm \mathrm{SD} ; * * \mathrm{p}<0.01$ vs. SUPINE; ${ }^{\dagger} \mathrm{p}<0.01$ vs. SEATED; ${ }^{\dagger} \mathrm{p}<0.05,{ }^{\dagger \dagger} \mathrm{p}<0.01$ vs. 0-10; $\mathrm{VLF}$, very low frequency; $\dot{\mathrm{V}}_{\mathrm{E}}$, ventilation; $\dot{\mathrm{V}} \mathrm{O}_{2}$, oxygen uptake. 
Table 6 Heart rate variability and respiratory variables for male and female participants during rest.

\begin{tabular}{|c|c|c|}
\hline & $\begin{array}{c}\text { Male } \\
(n=14)\end{array}$ & $\begin{array}{c}\text { Female } \\
(n=16)\end{array}$ \\
\hline HR (bpm) & $64.7 \pm 12.6$ & $73.0 \pm 17.3^{\dagger}$ \\
\hline SDNN (ms) & $93.5 \pm 37.2$ & $75.6 \pm 33.3 *$ \\
\hline $\mathrm{SD} \Delta \mathrm{NN}(\mathrm{ms})$ & $71.0 \pm 42.1$ & $62.3 \pm 50.0$ \\
\hline RMSSD (ms) & $70.8 \pm 41.8$ & $62.7 \pm 49.9$ \\
\hline SD1 (ms) & $50.2 \pm 29.7$ & $44.0 \pm 35.4$ \\
\hline $\mathrm{SD} 2(\mathrm{~ms})$ & $121.3 \pm 46.0$ & $95.7 \pm 36.1 *$ \\
\hline $\mathrm{TP}\left(\mathrm{ms}^{2}\right)$ & $9681 \pm 7459$ & $6627 \pm 7337^{*}$ \\
\hline $\mathrm{VLF}\left(\mathrm{ms}^{2}\right)$ & $3873 \pm 3771$ & $2076 \pm 1762^{*}$ \\
\hline $\mathrm{LF}\left(\mathrm{ms}^{2}\right)$ & $3186 \pm 2762$ & $1782 \pm 2060 * *$ \\
\hline $\mathrm{LF}(\mathrm{nu})$ & $61.62 \pm 19.04$ & $50.54 \pm 22.50^{*}$ \\
\hline $\mathrm{HF}\left(\mathrm{ms}^{2}\right)$ & $2422 \pm 2724$ & $2629 \pm 5210$ \\
\hline $\mathrm{HF}(\mathrm{nu})$ & $35.83 \pm 18.96$ & $45.96 \pm 22.17^{*}$ \\
\hline $\mathrm{LF} / \mathrm{HF}$ & $3.50 \pm 4.82$ & $2.00 \pm 2.26^{*}$ \\
\hline $\mathrm{TV}(\mathrm{L})$ & $0.86 \pm 0.38$ & $0.54 \pm 0.21 * * *$ \\
\hline RR (breaths $\cdot \min ^{-1}$ ) & $13.50 \pm 3.96$ & $14.99 \pm 3.54$ \\
\hline$\dot{\mathrm{V}}_{\mathrm{E}}\left(\mathrm{L} \cdot \mathrm{min}^{-1}\right)$ & $8.94 \pm 2.41$ & $6.54 \pm 1.96^{* * *}$ \\
\hline$\dot{\mathrm{V}} \mathrm{O}_{2}\left(\mathrm{~mL} \cdot \mathrm{kg}^{-1} \cdot \mathrm{min}^{-1}\right)$ & $4.54 \pm 1.09$ & $3.88 \pm 1.19 *$ \\
\hline
\end{tabular}

Values are mean $\pm \mathrm{SD} ;{ }^{\ddagger} \mathrm{p} \leq 0.06, * \mathrm{p}<0.05, * * \mathrm{p}<0.01, * * * \mathrm{p}<0.001$ vs. Male; HR, heart rate; SDNN, standard deviation of RR (NN) intervals; SD $\triangle N N$, standard deviation of the change in RR intervals; RMSSD, square root of the mean squared differences of successive RR intervals; SD1, standard deviation of instantaneous RR variability; SD2, standard deviation of long-term RR variability; TP, total power; VLF, very low frequency; LF, low frequency; nu, normalised units; HF, high frequency; $\mathrm{LF} / \mathrm{HF}$, low frequency to high frequency ratio; TV, tidal volume; RR, respiratory rate; $\dot{\mathrm{V}}_{\mathrm{E}}$, ventilation; $\dot{\mathrm{V}} \mathrm{O}_{2}$, oxygen uptake. 
Table 7 Reliability measures including intraclass correlation coefficients $(\mathrm{CV}, \%)$ for consecutive 10-minute heart rate variability and respiratory variables for male and female participants during supine, seated and standing rest ( $\mathrm{n}=14$ male, $\mathrm{n}=16$ female).

\begin{tabular}{|c|c|c|c|c|c|c|}
\hline & \multicolumn{2}{|c|}{ Supine } & \multicolumn{2}{|c|}{ Seated } & \multicolumn{2}{|c|}{ Standing } \\
\hline & Male & Female & Male & Female & Male & Female \\
\hline HR (bpm) & $0.911(2.8)$ & $0.980(2.4)$ & $0.913(3.1)$ & $0.942(3.0)$ & $0.967(2.4)$ & $0.946(3.6)$ \\
\hline SDNN (ms) & $0.813(15.5)$ & 0.851 (11.6) & 0.899 (11.9) & $0.712(13.9)$ & $0.897(12.3)$ & $0.731(11.2)$ \\
\hline $\mathrm{SD} \Delta \mathrm{NN}(\mathrm{ms})$ & $0.925(11.2)$ & $0.956(8.9)$ & $0.886(12.5)$ & 0.808 (15.6) & $0.943(13.1)$ & $0.640(16.8)$ \\
\hline RMSSD (ms) & $0.922(11.4)$ & $0.956(8.9)$ & $0.886(12.4)$ & $0.809(15.6)$ & $0.943(13.1)$ & $0.368(23.8)$ \\
\hline SD1 (ms) & $0.927(11.2)$ & $0.956(8.9)$ & $0.886(12.5)$ & $0.808(15.6)$ & $0.943(13.1)$ & $0.640(16.7)$ \\
\hline $\mathrm{SD} 2$ (ms) & $0.744(17.9)$ & $0.739(14.1)$ & $0.895(12.8)$ & $0.670(14.7)$ & $0.887(12.4)$ & $0.733(11.2)$ \\
\hline $\mathrm{TP}\left(\mathrm{ms}^{2}\right)$ & $0.828(29.5)$ & $0.830(24.3)$ & $0.868(28.4)$ & $0.700(32.1)$ & $0.889(27.8)$ & $0.620(28.5)$ \\
\hline $\operatorname{VLF}\left(\mathrm{ms}^{2}\right)$ & $0.731(46.0)$ & $0.098 *(53.6)$ & $0.808(43.2)$ & $0.358(45.9)$ & $0.839(34.9)$ & $0.583(34.8)$ \\
\hline $\mathrm{LF}\left(\mathrm{ms}^{2}\right)$ & $0.729(34.6)$ & $0.864(32.5)$ & $0.825(35.8)$ & 0.734 (39.5) & $0.829(30.8)$ & $0.587(35.1)$ \\
\hline $\mathrm{LF}(\mathrm{nu})$ & 0.699 (19.9) & $0.865(24.0)$ & $0.651(15.3)$ & $0.709(21.3)$ & $0.819(7.1)$ & $0.723(10.6)$ \\
\hline $\mathrm{HF}\left(\mathrm{ms}^{2}\right)$ & $0.820(29.9)$ & $0.931(20.7)$ & $0.819(36.4)$ & $0.734(38.4)$ & $0.933(31.7)$ & $0.546(40.7)$ \\
\hline $\mathrm{HF}(\mathrm{nu})$ & $0.718(17.3)$ & $0.858(12.0)$ & $0.664(25.4)$ & $0.724(19.3)$ & $0.843(19.1)$ & $0.721(26.9)$ \\
\hline $\mathrm{LF} / \mathrm{HF}$ & $0.721(38.2)$ & 0.854 (35.7) & $0.731(43.8)$ & 0.703 (41.4) & $0.888(25.7)$ & $0.738(36.3)$ \\
\hline TV (L) & $0.881(8.8)$ & $0.902(8.1)$ & $0.962(7.3)$ & $0.942(10.5)$ & $0.954(6.7)$ & $0.912(9.1)$ \\
\hline RR (breaths $\cdot \min ^{-1}$ ) & $0.944(8.0)$ & $0.901(6.6)$ & $0.943(6.1)$ & $0.877(7.4)$ & $0.965(5.3)$ & $0.909(6.1)$ \\
\hline$\dot{\mathrm{V}}_{\mathrm{E}}\left(\mathrm{L} \cdot \mathrm{min}^{-1}\right)$ & $0.909(8.4)$ & $0.861(6.3)$ & $0.850(7.9)$ & $0.902(11.0)$ & $0.918(6.5)$ & $0.922(8.2)$ \\
\hline$\dot{\mathrm{V}} \mathrm{O}_{2}\left(\mathrm{ml} \cdot \mathrm{kg}^{-1} \cdot \mathrm{min}^{-1}\right)$ & $0.932(5.9)$ & $0.855(5.9)$ & $0.899(6.0)$ & $0.923(9.8)$ & $0.895(6.3)$ & $0.932(9.0)$ \\
\hline
\end{tabular}

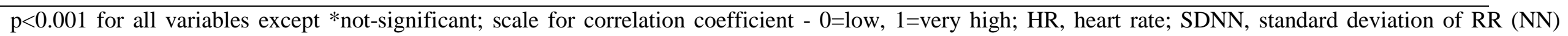

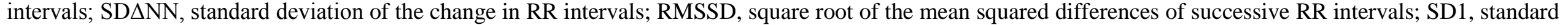

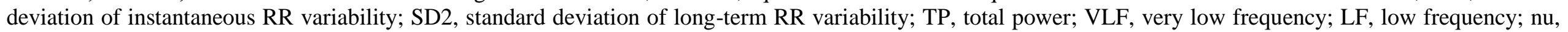
normalised units; LF/HF, low frequency to high frequency ratio; TV, tidal volume; RR, respiratory rate; $\dot{\mathrm{V}}_{\mathrm{E}}$, ventilation. $\dot{\mathrm{V}} \mathrm{O}_{2}$, oxygen uptake. 
Figure 1 Stability of consecutive 10-minute heart rate variability measures during 40-minutes of rest $(n=30)$. HR heart rate; SDNN, standard deviation of RR $(N N)$ intervals; SD $\triangle N N$, standard deviation of the change in RR intervals; RMSSD, square root of the mean squared differences of successive RR intervals; SD1, standard deviation of instantaneous RR variability; SD2, standard deviation of long-term RR variability; TP, total power; VLF, very low frequency; LF, low frequency; nu, normalised units; HF, high frequency; LF/HF, low frequency to high frequency ratio. 
